\title{
LINEAR RECEIVERS FOR MASSIVE MIMO FBMC/OQAM UNDER STRONG CHANNEL FREQUENCY SELECTIVITY
}

\author{
Xavier Mestre*, François Rottenberg ${ }^{\dagger}$, Mònica Navarro* \\ ${ }^{*}$ Centre Tecnològic de Telecomunicacions de Catalunya (Spain) \\ ${ }^{\dagger}$ Université Catholique de Louvain (Belgium)
}

\begin{abstract}
Filterbank Multicarrier (FBMC) modulations based on OQAM (FBMC/OQAM) have become a promising alternative to conventional OFDM because of their higher spectral efficiency and their improved selectivity in the frequency domain. Unfortunately, the orthogonality of these modulations is lost when the channel presents strong frequency selectivity, meaning that the channel response cannot be approximated as frequency flat within each subcarrier bandwidth. In this paper, this effect is analyzed in the massive MIMO setting, whereby the number of transmit and receive antennas is asymptotically large (but not as large as the number of subcarriers). It is formally shown that, under these asymptotic conditions, the output mean squared error (MSE) at each subcarrier converges to a constant independent of the subcarrier index. This was previously referred to as "self-equalization" principle in the FBMC/OQAM literature. It is demonstrated here that this phenomenon is a direct consequence of channel hardening effect in large scale MIMO configurations.
\end{abstract}

Index Terms - FBMC/OQAM, Massive MIMO, Random Matrix Theory.

\section{INTRODUCTION}

The increasing demand for high data rates has motivated the recent interest in novel spectrally efficient multicarrier waveforms. These new modulations improve the spectral efficiency of cyclic prefix based OFDM while maintaining most of the advantages of multicarrier signalling. Among the wide range of proposed alternatives, FBMC signals based on offset QAM (i.e. FBMC/OQAM) $[1,2,3]$ achieve the optimum spectral efficiency and can be implemented with very selective pulse shaping filters, reducing the need for guard bands. On the downside, FBMC/OQAM has some specific features that prevent direct application of conventional MIMO processing techniques, such as the fact that orthogonality is only achieved in the real domain and under frequency flat per-subcarrier channel conditions $[4,5]$. Several architectures have been proposed in the literature in order to alleviate the effect of high channel frequency selectivity, e.g. multi-tap $[6,7]$, multi-filterbank $[8,9]$ or frequency domain processing $[10,11]$. All these techniques achieve a very good performance, at the expense of a substantial increase in computational cost.

The publication of this paper has been partly supported by the Catalan and Spanish grants 2014SGR1567 and TEC2014-59255-C3-1. Part of this work has been performed in the framework of the Horizon 2020 project FANTASTIC-5G (ICT-671660), which is partly funded by the European Union. The authors would like to acknowledge the contributions of their colleagues in FANTASTIC-5G.
In parallel with this, massive MIMO (mMIMO) techniques are nowadays being considered as a natural means for boosting the data rate without the need for new spectrum availability $[12,13]$. By using a very large number of antennas at both the transceiver and the receiver, these systems are able to support a large number of spatially multiplexed transmissions that employ the same frequency band. Unfortunately, due to the high number of antenna elements involved in mMIMO transceiver, the combination with FBMC/OQAM under strong channel frequency selectivity conditions appears far from trivial. Note that conventional MIMO processing (based on multi-tap, multi-filterbank or frequency domain equalization) becomes computationally unaffordable as the number of antenna ports grows large. For this reason, conventional linear processing per-subcarrier appears to be the only viable alternative in large scale MIMO configurations.

Some recent contributions $[14,15,16]$ have pointed out that MIMO per-subcarrier processing of FBMC/OQAM signals can in fact benefit from the presence of a large number of antennas. Simulation results in [14] corroborate this point, which indicates that the channel hardening effect in mMIMO is inherently associated to a self-equalization effect of the received signal. The objective of this paper is to explore this phenomenon and characterize the effect of channel frequency selectivity in terms of the output MSE. Our analysis follows a two step asymptotic approach. First, we characterize the distortion at the output of the linear receivers assuming that the number of subcarriers is large. Then, the resulting MSE is analyzed by considering randomly generated finite impulse response channels and letting the number of transmit and receive antennas grow to infinity at the same rate. Results will therefore characterize a situation where all these quantities are large, but the number of subcarriers is much larger than the number of transmit/receive antennas. Note that this is indeed the situation in conventional wireless systems, where the number of subcarriers is typically around several thousands, whereas the number of antennas may only be as large as a few hundreds at most.

\section{FBMC/OQAM SYSTEM AND LINEAR RECEIVERS}

Assume an FBMC/OQAM modulation transceiver with $2 M$ subcarriers that employs a prototype pulse denoted by $p[n]$ at both the transmit (synthesis) and receive (analysis) filterbanks. Assume that this pulse has length $2 M \kappa$ ( $\kappa$ being the overlapping factor), even symmetric in the time domain and perfect reconstruction (PR) compliant. In addition to this, assume that the pulse is obtained as a discretization of a smooth $\mathcal{C}^{2}$ waveform $p(t):[-\kappa / 2, \kappa / 2] \rightarrow \mathbb{R}$, and that this waveform and its first and second order derivatives null out at the end points of its support. We will refer to $p^{(r)}[n]$ as the 
discretization of the $r$ th derivative of the analog waveform $p^{(r)}(t)$.

Consider the transmission of $N_{T}$ parallel OQAM modulated symbols per subcarrier (one per transmit antenna, using conventional spatial multiplexing). The OQAM symbols transmitted at the $m$ th subcarrier are denoted by a column vector $\mathbf{d}_{m}[n] \in \mathbb{R}^{N_{T} \times 1}$. These symbol streams are converted into $N_{T}$ FBMC/OQAM-modulated signals that are sent through each of the $N_{T}$ transmit antennas, which may by collocated or distributed (e.g. in a multi-user context). These signals go through a frequency selective MIMO channel and received by a single equipment with $N_{R}$ antenna ports. Let $\mathbf{H}(\omega) \in$ $\mathbb{C}^{N_{R} \times N_{T}}$ denote the MIMO channel frequency response matrix. The signal received at each of the $N_{R}$ antennas goes through an analysis filterbank, so that the output associated with the $k$ th subcarrier will be denoted by $\mathbf{y}_{k}[n] \in \mathbb{C}^{N_{R} \times 1}$. At the output of the analysis filterbanks corresponding to the $k$ th subcarrier, the receiver applies a linear transformation $\mathbf{B}_{k} \in \mathbb{C}^{N_{T} \times N_{R}}$ in order to retrieve the originally transmitted symbols. The actual symbols are then estimated by taking the real part of the output samples, namely [3]:

$$
\hat{\mathbf{d}}_{k}[n]=\operatorname{Re}\left[\mathbf{B}_{k} \mathbf{y}_{k}[n]\right] .
$$

Ideally we would like to have $\hat{\mathbf{d}}_{k}[n]=\mathbf{d}_{k}[n]$ for $k=1, \ldots, 2 M$. In practice, however, due to the presence of noise and the channel frequency selectivity, the output of the receive matrix contains a distorted version of the originally transmitted symbols, in the sense that

$$
\hat{\mathbf{d}}_{k}[n]=\operatorname{Re}\left[\mathbf{B}_{k} \mathbf{H}_{k}\right] \mathbf{d}_{k}[n]+\mathbf{e}_{k}[n]+\operatorname{Re}\left[\mathbf{B}_{k} \mathbf{w}_{k}[n]\right]
$$

where $\mathbf{e}_{k}[n]$ and $\mathbf{w}_{k}[n]$ respectively contain the contribution from inter-symbol/inter-carrier interference (ISI/ICI) and background noise, which is modeled as a circularly symmetric random variable with zero mean and power $\sigma^{2}$. Assuming that the symbols $\mathbf{d}_{k}[n]$ have power $P_{s}$, we can define the total MSE associated with the $k$ th subcarrier as

$$
\mathbb{E}\left\|\hat{\mathbf{d}}_{k}[n]-\mathbf{d}_{k}[n]\right\|^{2}=P_{e}(k)+\frac{\sigma^{2}}{2} \operatorname{tr}\left[\mathbf{B}_{k}^{H} \mathbf{B}_{k}\right]
$$

where $P_{e}(k)$ is the ISI/ICI distortion power at the $k$ th subcarrier, defined as

$$
P_{e}(k)=\mathbb{E}\left[\left\|\operatorname{Re}\left[\mathbf{B}_{k} \mathbf{H}_{k}-\mathbf{I}\right] \mathbf{d}_{k}[n]+\mathbf{e}_{k}[n]\right\|^{2}\right] .
$$

This distortion power is generally difficult to characterize due to the complicated structure of the FBMC/OQAM signal. However, under certain regularity conditions, one can obtain a very accurate approximation by assuming an asymptotically large number of subcarriers $(2 M)$.

Assume that the linear receiver applied at the $k$ th subcarrier is obtained as $\mathbf{B}_{k}=\mathbf{B}\left(\omega_{k}\right)$ where $\omega_{k}=\pi k / M$ is the radial frequency associated with the $k$ th subcarrier, and where $\mathbf{B}(\omega)$ is a smooth function of $\omega$ on $\mathbb{R} / 2 \pi \mathbb{Z}$. Assume that $\mathbf{H}(\omega)$ is also a smooth function on $\mathbb{R} / 2 \pi \mathbb{Z}$ and let $\mathbf{H}_{k}=\mathbf{H}\left(\omega_{k}\right)$ and $\mathbf{H}_{k}^{(\ell)}=$ $\mathbf{H}^{(\ell)}\left(\omega_{k}\right)$ where $\mathbf{H}^{(\ell)}(\omega)$ is the $\ell$ th derivative of the channel frequency response. Assuming that the original symbols are independent and identically distributed (i.i.d.) bounded random variables with zero mean and power $P_{s} / 2$, we can write $P_{e}(k)=\bar{P}_{e}(k)+$ $o\left(M^{-2}\right)$ as $M \rightarrow \infty$, where $\bar{P}_{e}(k)$ is defined as [9]

$$
\begin{aligned}
\bar{P}_{e}(k) & =\frac{P_{s}}{2} \operatorname{tr}\left[\left(\mathbf{B}_{k} \mathbf{H}_{k}-\mathbf{I}_{N_{T}}\right)\left(\mathbf{B}_{k} \mathbf{H}_{k}-\mathbf{I}_{N_{T}}\right)^{H}\right] \\
& +P_{s} \frac{\eta}{(2 M)^{2}} \operatorname{Re} \operatorname{tr}\left[\left(\mathbf{B}_{k} \mathbf{H}_{k}-\mathbf{I}_{N_{s}}\right)\left(\mathbf{H}_{k}^{(2)}\right)^{H} \mathbf{B}_{k}^{H}\right] \\
& +P_{s} \frac{\eta}{(2 M)^{2}} \operatorname{tr}\left[\mathbf{B}_{k} \mathbf{H}_{k}^{(1)}\left(\mathbf{H}_{k}^{(1)}\right)^{H} \mathbf{B}_{k}^{H}\right]
\end{aligned}
$$

and where $\eta$ is a positive quantity that depends on the prototype pulse $p[n]$ as follows. Let $\mathbf{P} \in \mathbb{R}^{2 M \times \kappa}$ and $\mathbf{D} \in \mathbb{R}^{2 M \times \kappa}$ denote two matrices obtained by respectively arranging the $2 M \kappa$ samples of the prototype pulse $p[n]$ and its derivative $p^{(1)}[n]$ in columns. Let $\mathcal{R} \in \mathbb{R}^{2 M \times 2 \kappa-1}$ and $\mathcal{S} \in \mathbb{R}^{2 M \times 2 \kappa-1}$ denote the two matrices:

$$
\begin{aligned}
\mathcal{R} & =\mathbf{P} \circledast \mathbf{J}_{2 M} \mathbf{D} \\
\mathcal{S} & =\left(\mathbf{J}_{2} \otimes \mathbf{I}_{M}\right) \mathbf{P} \circledast \mathbf{J}_{2 M} \mathbf{D}
\end{aligned}
$$

where $\circledast$ denotes row-wise convolution (providing a matrix with rows equal to the convolution of the rows of the two arguments), $\otimes$ is the Kronecker product and $\mathbf{J}_{k}$ is the $k \times k$ exchange matrix (i.e. an all zero matrix with ones in the anti-diagonal). The pulse-specific quantity $\eta$ is thereby defined as

$$
\eta=\frac{1}{2 M} \operatorname{tr}\left[\mathbf{U}^{+} \mathcal{R} \mathcal{R}^{T}+\mathbf{U}^{-} \mathcal{S S}^{T}\right]
$$

where $\mathbf{U}^{ \pm}=\mathbf{I}_{2} \otimes\left(\mathbf{I}_{M} \pm \mathbf{J}_{M}\right)$.

Consider the asymptotic expression of the MSE, namely

$$
\operatorname{MSE}(k)=\overline{P_{e}}(k)+\frac{\sigma^{2}}{2} \operatorname{tr}\left[\mathbf{B}_{k}^{H} \mathbf{B}_{k}\right] .
$$

We can try to design the linear receiver $\mathbf{B}_{k}$ so as to minimize the MSE at the $k$ th subcarrier according to (2). Unfortunately, this problem is generally difficult to solve (see [17] for further details), so that it is more conventional to design the receiver in order to minimize the MSE under frequency flat channel conditions per subcarrier, namely

$$
\begin{aligned}
\operatorname{MSE}_{\text {flat }}(k)= & \frac{P_{s}}{2} \operatorname{tr}\left[\left(\mathbf{B}_{k} \mathbf{H}_{k}-\mathbf{I}_{N_{T}}\right)\left(\mathbf{B}_{k} \mathbf{H}_{k}-\mathbf{I}_{N_{T}}\right)^{H}\right] \\
& +\frac{\sigma^{2}}{2} \operatorname{tr}\left[\mathbf{B}_{k}^{H} \mathbf{B}_{k}\right] .
\end{aligned}
$$

The receiver that minimizes the above cost function can be expressed as

$$
\mathbf{B}_{k}^{L M M S E}=\left(\mathbf{H}_{k}^{H} \mathbf{H}_{k}+\sigma^{2} / P_{s} \mathbf{I}_{N_{T}}\right)^{-1} \mathbf{H}_{k}^{H} .
$$

The construction of this receiver implies the knowledge of the noise power. An alternative receiver when this quantity is unknown is the zero forcing (ZF) filter, namely $\mathbf{B}_{k}^{Z F}=\left(\mathbf{H}_{k}^{H} \mathbf{H}_{k}\right)^{-1} \mathbf{H}_{k}^{H}$, which is only implementable if $N_{T} \leq N_{R}$. Sometimes it is convenient in massive MIMO architectures to avoid the channel inversion. In these situations, one may resort to the matched filter (MF), which in our case will be defined as $\mathbf{B}_{k}^{M F}=N_{T}^{-1} \mathbf{H}_{k}^{H}$.

The large $M$ performance of these three receivers can be evaluated in terms of MSE by directly replacing the expression of the filter into the definition of (1), and using the asymptotic distortion power $\bar{P}_{e}(k)$ as defined above. It will be shown below that in practice this approximation turns out to be extremely good, even for relatively low values of the number of subcarriers.

\section{MASSIVE MIMO PERFORMANCE}

In order to investigate the performance of the above linear receivers in a massive MIMO configuration, we consider a random model for the MIMO channel frequency response. More specifically, we assume that the channel $\mathbf{H}(\omega)$ is modeled as an $L$ tap finite impulse response channel with a Kronecker correlation model, i.e.

$$
\begin{aligned}
\mathbf{H}(\omega) & =\sum_{\ell=0}^{L-1} \sqrt{p_{\ell}} \mathbf{C}_{R}^{1 / 2} \mathbf{G}_{\ell} \mathbf{C}_{T}^{1 / 2} \mathrm{e}^{-\mathrm{j}(\ell-1) \omega} \\
& =\left(\mathbf{f}^{H}(\omega) \otimes \mathbf{C}_{R}^{1 / 2}\right) \mathbf{G} \mathbf{C}_{T}^{1 / 2}
\end{aligned}
$$


where each $\mathbf{G}_{\ell}$ is a matrix with i.i.d. entries following a circularly symmetric complex Gaussian distribution with zero mean and unit variance, $p_{\ell}$ is the power associated to the $\ell$ th tap,

$$
\begin{aligned}
\mathbf{G} & =\left[\mathbf{G}_{0}^{T}, \ldots, \mathbf{G}_{L-1}^{T}\right]^{T} \\
\mathbf{f} & =\left[\sqrt{p_{0}}, \sqrt{p_{1}} \mathrm{e}^{\mathrm{j} \omega}, \ldots, \sqrt{p_{L-1}} \mathrm{e}^{\mathrm{j}(L-1) \omega}\right]^{T}
\end{aligned}
$$

and where $\mathbf{C}_{R}$ and $\mathbf{C}_{T}$ are the spatial correlation matrices at the transmitter and the receiver, respectively. We will normalize the channel energy so that $N_{T}^{-1} \operatorname{tr}\left[\mathbf{C}_{T}\right]=N_{R}^{-1} \operatorname{tr}\left[\mathbf{C}_{R}\right]=\|\mathbf{f}\|=1$ and let $P_{s}=P_{T} / N_{T}$, so that the total transmitted power is constant regardless of the number of transmit antennas. The objective of this paper is to characterize the asymptotic performance of the above receivers when both the number of transmit and receive antennas grows without bound. We will assume that the number of subcarriers is much larger than the number of transmit/receive antennas (namely $\max \left(N_{T}, N_{R}\right) / M \rightarrow 0$ ), so that we can take $\bar{P}_{e}(k)$ as the actual distortion power at the output of the linear receiver.

Let $\hat{\mathbf{Q}}_{k}(x) \in \mathbb{C}^{N_{T} \times N_{T}}$ denote a matrix-valued random function of $x \in \mathbb{R}^{+}$defined as

$$
\hat{\mathbf{Q}}_{k}(x)=\left(\frac{1}{N_{T}} \mathbf{G}^{H} \mathbf{C}_{R}^{(k)} \mathbf{G}+x \mathbf{C}_{T}^{-1}\right)^{-1}
$$

where

$$
\mathbf{C}_{R}^{(k)}=\mathbf{f}\left(\omega_{k}\right) \mathbf{f}^{H}\left(\omega_{k}\right) \otimes \mathbf{C}_{R}
$$

It can be seen from (2) that we can essentially express the MSE at the $k$ th subcarrier in terms of functions of $\hat{\mathbf{Q}}_{k}(x)$. In order to describe the asymptotic behavior of this matrix, it is useful to define the following pair of deterministic matrices for $x \in \mathbb{R}^{+}$:

$$
\begin{aligned}
\mathbf{Q}(x) & =\mathbf{C}_{T}\left(\tilde{\delta}(x) \mathbf{C}_{T}+x \mathbf{I}_{N_{T}}\right)^{-1} \\
\widetilde{\mathbf{Q}}_{k}(x) & =\mathbf{C}_{R}^{(k)}\left(\delta(x) \mathbf{C}_{R}^{(k)}+\frac{N_{T}}{L N_{R}} \mathbf{I}_{L N_{R}}\right)^{-1}
\end{aligned}
$$

where the pair $\delta(x), \tilde{\delta}(x)$ are the unique positive solutions to the system of two equations

$$
\tilde{\delta}(x)=\frac{1}{L N_{R}} \operatorname{tr}\left[\widetilde{\mathbf{Q}}_{k}(x)\right] \quad \delta(x)=\frac{1}{L N_{R}} \operatorname{tr}[\mathbf{Q}(x)] .
$$

It can readily be seen that the eigenvalues of $\widetilde{\mathbf{Q}}_{k}(x)$ do not depend on $k$, and therefore both $\tilde{\delta}(x)$ and $\delta(x)$ are constant for all subcarriers. The deterministic matrix-valued function $\mathbf{Q}(x)$ is typically referred to as the asymptotic equivalent of the random $\hat{\mathbf{Q}}_{k}(x)$. This is because (under certain conditions that will be specified later) one can show that $N_{T}^{-1}\left|\operatorname{tr}\left[\mathbf{A} \hat{\mathbf{Q}}_{k}(x)\right]-\operatorname{tr}[\mathbf{A Q}(x)]\right| \rightarrow 0$ almost surely when $N_{T}, N_{R}$ tend to infinity at the same rate, where $\mathbf{A}$ is any deterministic matrix of bounded spectral norm. This fact will be fundamental in the characterization of the asymptotic MSE of the three receivers under consideration. We will also need some additional functions of $\widetilde{\mathbf{Q}}_{k}(x)$ and $\mathbf{Q}(x)$, namely

$$
\tilde{\gamma}(x)=\frac{1}{L N_{R}} \operatorname{tr}\left[\widetilde{\mathbf{Q}}_{k}^{2}(x)\right] \quad \gamma(x)=\frac{1}{L N_{R}} \operatorname{tr}\left[\mathbf{Q}^{2}(x)\right]
$$

together with

$$
\begin{aligned}
& \alpha(x)=\frac{1}{L N_{R}} \operatorname{tr}\left[\mathbf{C}_{T}\left(\tilde{\delta}(x) \mathbf{C}_{T}+x \mathbf{I}_{N_{T}}\right)^{-2}\right] \\
& \tilde{\alpha}(x)=\frac{1}{L N_{R}} \operatorname{tr}\left[\mathbf{C}_{R}^{(k)}\left(\delta(x) \mathbf{C}_{R}^{(k)}+\frac{N_{T}}{L N_{R}} \mathbf{I}_{L N_{R}}\right)^{-2}\right] .
\end{aligned}
$$

The following proposition shows that when both the number of transmit and receive antennas increases without bound, the random $\operatorname{MSE}(k)$ has the same asymptotic behavior as a quantity $\overline{\mathrm{MSE}}$ that does not depend on the subcarrier index $k$. The proof is based on standard random matrix theory techniques based on Gaussian tools and is omitted due to space constraints.

Proposition 1 Assume that the eigenvalues of $\mathbf{C}_{R}^{(k)}$ and $\mathbf{C}_{T}^{-1}$ are bounded, and that $N_{T}, N_{R} \rightarrow \infty$ such that the quotient $N_{T} / N_{R}$ is confined to a compact interval of $\mathbb{R}^{+}$. For the three receivers under consideration, the MSE defined in (2) is asymptotically equivalent to a deterministic quantity MSE independent of the subcarrier index, in the sense that $|\operatorname{MSE}(k)-\overline{\mathrm{MSE}}| \rightarrow 0$ almost surely. The value of $\overline{\mathrm{MSE}}$ is different for the three receivers under consideration. For the LMMSE receiver, we have

$$
\begin{gathered}
\overline{\operatorname{MSE}}_{L M M S E}=\frac{P_{T}}{2}\left(1-\frac{L N_{R}}{N_{T}} \delta(\lambda) \tilde{\delta}(\lambda)\right) \\
+\frac{P_{T} \eta}{(2 M)^{2}}\left[\operatorname{AD}^{2} \frac{L N_{R}}{N_{T}} \delta(\lambda) \tilde{\delta}(\lambda)+\mathrm{DS}^{2} \frac{(\tilde{\gamma}(\lambda)+\lambda \tilde{\alpha}(\lambda)) \alpha(\lambda)}{1-\tilde{\gamma}(\lambda) \gamma(\lambda)}\right]
\end{gathered}
$$

where $\lambda=\sigma^{2} / P_{T}$ and where we have defined the delay spread (DS) and the average delay (AS) of the channel as

$$
\mathrm{DS}^{2}=\sum_{\ell=0}^{L-1} \ell^{2} p_{\ell}-\mathrm{AD}^{2}, \quad \mathrm{AD}=\sum_{\ell=0}^{L-1} \ell p_{\ell} .
$$

For the $Z F$ receiver (assuming $\lim \sup N_{T} / N_{R}<1$ ), we have

$$
\begin{aligned}
\overline{\mathrm{MSE}}_{Z F} & =\frac{\sigma^{2}}{2} \frac{[\tilde{\delta}(0)-\delta(0) \tilde{\gamma}(0)] \xi_{T}^{(-1)}}{\tilde{\delta}^{2}(0)(1-\tilde{\gamma}(0) \gamma(0))}+P_{T} \frac{\eta \mathrm{AD}^{2}}{(2 M)^{2}} \\
& +P_{T} \frac{\eta \mathrm{DS}^{2}}{(2 M)^{2}} \frac{N_{T}}{L N_{R}} \frac{\tilde{\gamma}(0) \xi_{T}^{(-1)}}{\tilde{\delta}^{2}(0)(1-\tilde{\gamma}(0) \gamma(0))}
\end{aligned}
$$

whereas for the MF receiver

$$
\begin{array}{r}
\overline{\mathrm{MSE}}_{M F}=\frac{P_{T}}{2}\left[1-2 \frac{N_{R}}{N_{T}}+\left(\frac{N_{R}}{N_{T}}\right)^{2} \xi_{T}^{(2)}+\left(\frac{N_{R}}{N_{T}}\right) \xi_{R}^{(2)}\right] \\
+\left[\frac{\sigma^{2}}{2}+\frac{P_{T} \eta}{(2 M)^{2}}\left[\mathrm{DS}^{2}\left(1-\xi_{T}^{(2)} \frac{N_{R}}{N_{T}}\right)+\mathrm{AD}^{2}\right]\right] \frac{N_{R}}{N_{T}}
\end{array}
$$

where we have defined, for $k \in \mathbb{Z}$, the quantities

$$
\xi_{T}^{(k)}=\frac{1}{N_{T}} \operatorname{tr}\left[\mathbf{C}_{T}^{k}\right] \quad \xi_{R}^{(k)}=\frac{1}{N_{R}} \operatorname{tr}\left[\mathbf{C}_{R}^{k}\right] .
$$

According to the above result, the asymptotic performance of the three linear receivers considered here is roughly dictated by the frequency selectivity of the channel measured through the average delay AD and delay spread DS as defined in (4). Hence, the same asymptotic performance is asymptotically observed in all subcarriers regardless of the channel realization, as further illustrated in the following subsection. It must be pointed out that the asymptotic expressions obtained in Proposition 1 have been obtained by first letting $M \rightarrow \infty$ and then $N_{T}, N_{R} \rightarrow \infty$. One should therefore be very cautious when analyzing the behavior of the above expressions, since in practice one should always have $N_{T}, N_{R}<<2 M$ in order for these quantities to make sense. Fortunately, this is typically the case in conventional systems even for relatively low values of $2 M$, as illustrated in what follows. 


\section{SIMULATIONS}

In order to assess the accuracy of the derived asymptotic approximations, we considered a FBMC/OQAM system with $2 M=512$ subcarrier where the prototype pulse was chosen as proposed in [18] (PHYDYAS pulse), with overlapping factor equal to $\kappa=3$. The channel was generated according to an Extended Typical Urban (ETU) model [19] with an intercarrier separation of $15 \mathrm{kHz}$. The transmit and receive correlation matrices were fixed as Toeplitz with $i$ th diagonal equal to $\rho^{|i|}$ where $\rho=0.5$ at the transmitter and $\rho=0.8$ at the receiver. Simulations considered an FBMC/OQAM transmission of 200 multicarrier symbols and a total of 100 different channel realizations.

Figure 1 represents the MSE obtained at the output of the linear receiver at each subcarrier for a specific MIMO channel realization. Apart from the simulated values (cross markers) we also represent the large $M$ asymptotic MSE as given by (2) (solid lines) and the large $N_{T}, N_{R}<<M$ approximation established in Proposition 1 above (dashed lines). Note that the simulated values are virtually indistinguishable from the large $M$ MSE approximation. Furthermore, as the number of antennas increases (lower plot) the measured MSE becomes uniform across all the spectrum as predicted by Proposition 1 presented above.
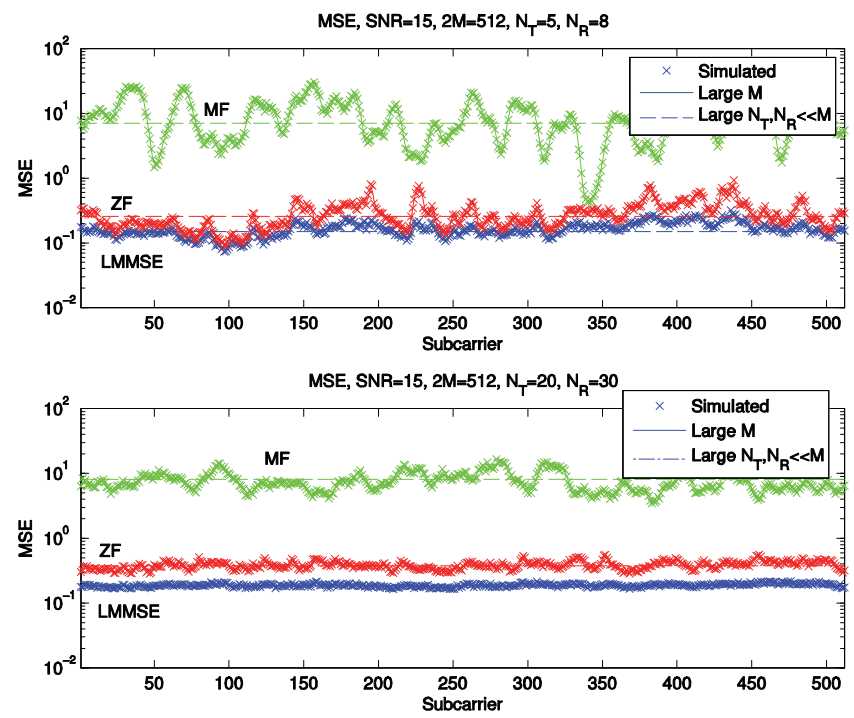

Fig. 1. Measured vs. asymptotic for a specific channel realization.

Figure 2 compares the simulated average MSE (averaged across subcarriers and channel realizations) with the asymptotic MSE described in Proposition 1 for different values of the number of transmit and receive antennas. Results are given in terms of average MSE as a function of the input signal to noise ratio (SNR), defined as $P_{T} / \sigma^{2}$. The vertical bars in these figures indicate the $90 \%$ confidence interval of the measured MSE across the spectrum and channel realizations. In other words, $90 \%$ of the subcarriers and channel realizations resulted in a simulated MSE within these vertical intervals. Observe that the asymptotic formulas provide a very accurate description of the average MSE even for relatively low values of $M, N_{T}$ and $N_{R}$. On the other hand, increasing the transmit and receive antennas we observe that the variance of the simulated MSE is substantially reduced, confirming the convergence as predicted by Proposition 1.

\section{CONCLUSIONS}

An asymptotic study of the performance of FBMC/OQAM in massive MIMO contexts has been provided. The performance is measured as MSE under the assumption of large number of subcarriers $(M)$ and then large number of transmit and receive antennas $\left(N_{T}\right.$, $N_{R}$ ). Hence, results are representative of a situation where all these parameters are large but $N_{T}, N_{R}<<M$, which is the case in conventional system configurations. Closed form expressions have been provided for the performance in terms of asymptotic MSE of the three conventional linear receivers, namely the LMMSE, the MF and the ZF receivers. It has been shown that, under these asymptotic conditions, the performance becomes uniform across the spectrum, a phenomenon that has been previously referred to as "selfequalization" in the literature.

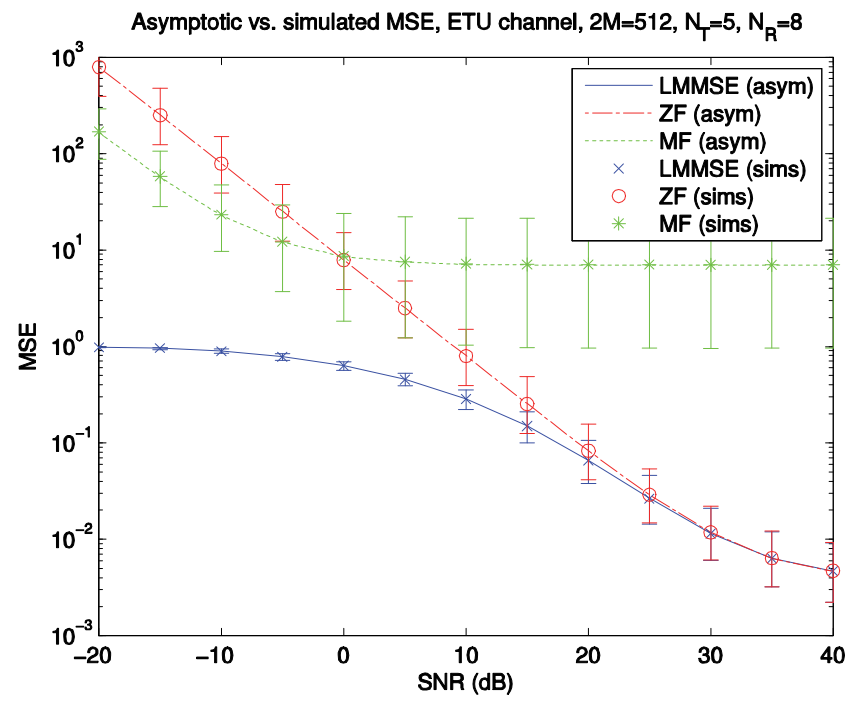

(a) $N_{T}=5$ and $N_{R}=8$

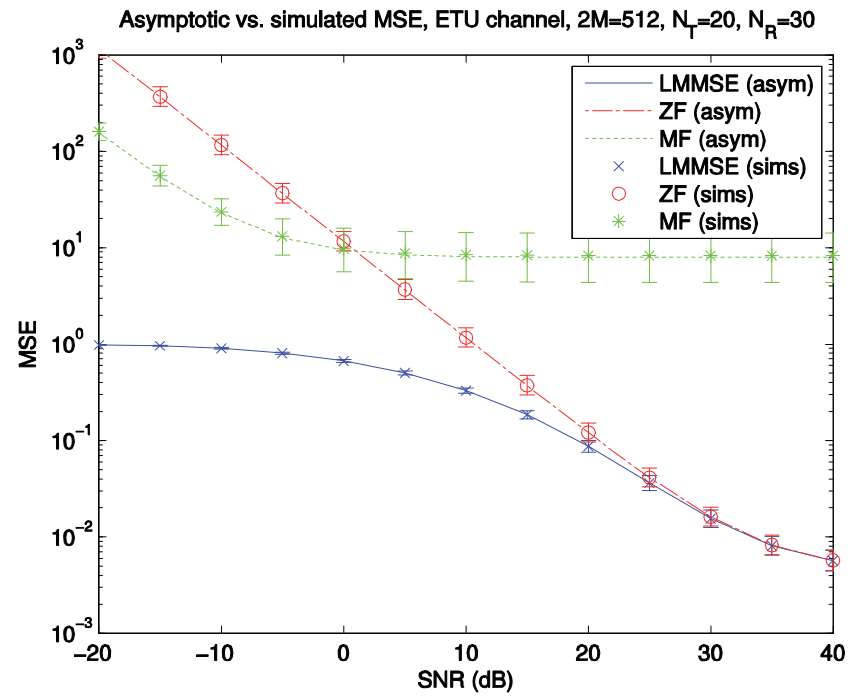

(b) $N_{T}=20$ and $N_{R}=30$

Fig. 2. Measured vs. asymptotic MSE in 100 channel realizations. Vertical bars represent the $90 \%$ confidence interval. 


\section{REFERENCES}

[1] B.R. Saltzberg, "Performance of an efficient parallel data transmission system," IEEE Transactions on Communication Technology, vol. 15, no. 6, pp. 805 - 811, Dec. 1967.

[2] B. Hirosaki, "An orthogonally multiplexed QAM system using the discrete Fourier transform," IEEE Transactions on Communications, vol. 29, no. 7, pp. 982-989, July 1981.

[3] P. Siohan, C. Siclet, and N. Lacaille, "Analysis and design of OFDM/OQAM systems based on filterbank theory," IEEE Transactions on Signal Processing, vol. 50, no. 5, pp. 1170 1183, May 2002.

[4] M Nájar, M. Payaró, E. Kofidis, M. Tanda, J. Louveaux, M. Renfors, T. Hidalgo, D. Le Ruyet, C. Lélé, R. Zacaria, and M. Bellanger, "MIMO techniques and beamforming," Deliverable D4.2, ICT PHYDYAS (PHYsical layer for DYnamic AccesS and cognitive radio) project, Feb. 2010.

[5] R. Zakaria, D. Le Ruyet, S. Nedic, X. Mestre, and L. Baltar, "Mimo transmission and reception design schemes," Deliverable D4.1, Enhanced Multicarrier Techniques for Professional Ad-Hoc and Cell-Based Communications (EMPhAtiC), September 2013.

[6] T. Ihalainen, T. Hidalgo Stitz, M. Rinne, and M. Renfors, "Channel equalization in filter bank based multicarrier modulation for wireless communications," EURASIP Journal on Advances in Signal Processing, vol. 2007, pp. 1-18, 2007.

[7] T. Ihalainen, A. Ikhlef, J. Louveaux, and M. Renfors, "Channel equalization for multi-antenna FBMC/OQAM receivers," IEEE Trans, on Vehicular Technology, vol. 60, no. 5, pp. 20702085, Jun. 2011.

[8] X. Mestre, M. Majoral, and S. Pfletschinger, "An asymptotic approach to parallel equalization of filter bank multicarrier signals," IEEE Transactions on Signal Processing, vol. 61, no. 14, pp. 3592-3606, July 2013.

[9] X. Mestre and D. Gregoratti, "Parallelized structures for MIMO FBMC under strong channel frequency selectivity," IEEE Transactions on Signal Processing, vol. 64, no. 5, pp. 1200-1215, Mar. 2016.

[10] M. Bellanger, "FS-FBMC: an alternative scheme for filter bank based multicarrier transmission," in Proceedings of the 5th International Symposium on Communications, Control and Signal Processing (ISCCSP), 2012.
[11] D. Mattera, M. Tanda, and M. Bellanger, "Frequencyspreading implementation of OFDM/OQAM systems," in Proceedings of the International Symposium on Wireless Communication Systems (ISWCS), 2012, pp. 176 - 180.

[12] T. Marzetta, "Noncooperative cellular wireless with unlimited numbers of base station antennas," IEEE Transactions on Wireless Communications, vol. 9, no. 11, pp. 3590-3600, Nov. 2010 .

[13] J. Hoydis, S. ten Brink, and M. Debbah, "Massive MIMO in the UL/DL of cellular networks: How many antennas do we need?," IEEE Journal on Selected Areas in Communications, vol. 31, no. 2, pp. 160-171, Feb. 2013.

[14] A. Farhang, N. Marchetti, L.E. Doyle, and B. FarhangBoroujeny, "Filter bank multicarrier for massive MIMO," in Proceedings of the IEEE 80th Vehicular Technology Conference (VTC Fall), 2014.

[15] A. Farhang, N. Marchetti, F. Figueiredo, and J.P. Miranda, "Massive MIMO and waveform design for 5th generation wireless communication systems," in Proceedings of the First International Conference on $5 G$ for Ubiquitous Connectivity (5GU), 2014, pp. 70-75.

[16] A. Aminjavaheri, A. Farhang, N. Marchetti, L. Doyle, and B. Farhang-Boroujeny, "Frequency spreading equalization in multicarrier massive MIMO," in Proceedings of the IEEE International Conference on Communication Workshop (ICCW), 2015, pp. $1292-1297$.

[17] F. Rottenberg, X. Mestre, and J Louveaux, "Optimal zero forcing precoder and decoder design for multiuser MIMO FBMC under strong channel selectivity," in Proceedings of the IEEE International Conference on Acoustics, Speech and Signal Processing, 2016.

[18] M.G. Bellanger, "Specification and design of a prototype filter for filter bank based multicarrier transmission," in Proceedings of the IEEE International Conference on Acoustics, Speech and Signal Processing, 2001, vol. 4, pp. 2417 - 2420.

[19] "LTE; evolved universal terrestrial radio access (e-UTRA); base station (BS) radio transmission and reception," Tech. Rep. TS 36.104 v11.4.0 release 11, 3GPP, 2013. 